\title{
Bézier Curve based Trajectory Planning for an Intelligent Wheelchair to Pass a Doorway
}

\author{
Ling Chen, Sen Wang, Huosheng Hu and Klaus McDonald-Maier \\ School of Computer Science and Electronic Engineering \\ University of Essex, Colchester CO4 3SQ, United Kingdom \\ E-mail: \{lcheno, swangi, hhu, kdm\}@essex.ac.uk
}

\begin{abstract}
Door passing is the basic capability of an intelligent wheelchair. This paper presents a novel approach to address the door passing issue using Bézier curve based trajectory planning. The planed path consists of two segments: one from corridor to door and the other from door to the goal position. For each segment, an optimal Bézier curve is generated as a reference trajectory for an intelligent wheelchair to travel smoothly and accurately subject to corridor constraints, curvature limitation and obstacles. The simulation is conducted to verify the feasibility of the proposed approach, and the results show a good performance in terms of tracking accuracy and good maneuverability.
\end{abstract}

Keywords-Door passing; Bézier curve; optimization; trajectory planning; obstacle avoidance

\section{INTRODUCTION}

Door passing is considered as the fundamental capability of intelligent wheelchairs that are operated in an indoor environment. The wheelchair users are normally elderly and disabled, who may suffer some types of disabilities such as Parkinson's disease. They have difficulty in operating wheelchair using a traditional joystick, not to mention to pass through the constrained doorway. Therefore, there is great demand that the intelligent wheelchair could autonomously travel through confined and narrow doorways without user intervention or carer supervision.

In general, this problem can be addressed by two typical methods - obstacle avoidance and trajectory planning. Among all the methodologies solving obstacle avoidance, potential field based method gains the most popularity [1][3]. Although it provides an elegant solution to the obstacle avoidance problem, it has the disadvantage of falling into a local minimum as well as being undulating in some singularity point which is unacceptable for the wheelchair, as human being sitting on the wheelchair will feel uncomfortable in this circumstance. Meanwhile, some existing wheelchairs provide the door passage mode, such as the TAO one [4]. However, to the best of our knowledge, they all use the range finder sensor, e.g., infrared, to detect the door frame and avoid it at a low moving speed and the curvature of the traveling trajectory is not smooth and continous [4].

Recently, a trajectory planning based method drives the wheelchair to follow a desired path. Due to the small ratio of the door width to the wheelchair width (only about 300 $\mathrm{mm}$ access space for the wheelchair [5], see Fig. 1), it is

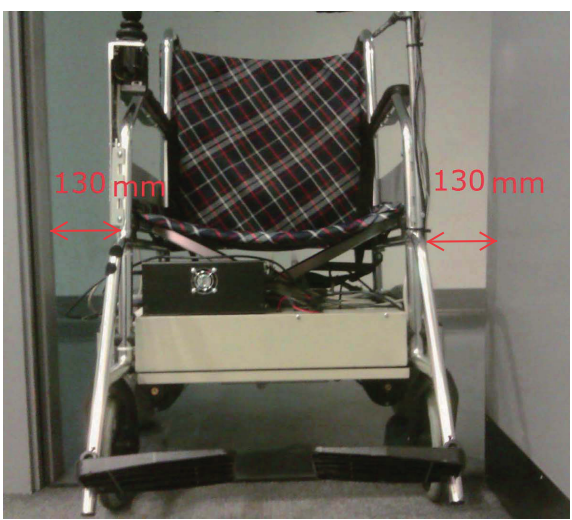

Fig. 1. Wheelchair in the doorway

difficult for the wheelchair to pass through the door at every heading direction. A frontier point method integrated with simultaneous localization and mapping was used for door passing of wheelchair in [6]. However, it needs to continuously generate the mean frontier points, which makes the curves not smooth and time consuming. A Case-Based Reasoning method for door passing was also presented in [7]. But it neither considered the smoothness of the trajectory nor the narrow structure of the doorway.

In order to produce a smooth trajectory, trajectory planning based on circular arc [8], spline [9] and Bézier curves [10], [11] can be adopted. Jolly et. al [10] proposed an efficient, Bézier curve based approach for the trajectory planning of a mobile robot, considering the boundary conditions, velocity limitation, etc. Choi et. al in [11] presented two trajectory planning algorithms based on Bézier curve for autonomous vehicles with constraints produced by waypoints and corridor width. However, it regards the vehicle as the particle, which is infeasible for the door passing of the wheelchair, in addition the obstacle avoidance has not been taken into account.

This paper intends to propose an efficient strategy for an intelligent wheelchair to pass through the narrow doorway in real time and with the greatest possible success. It introduces the Bézier curve based trajectory planning and the constrained optimization to generate smooth trajectories for the wheelchair to follow without significantly reducing the speed. Moreover, the proposed scheme can avoid the static obstacles while moving to the destination. 


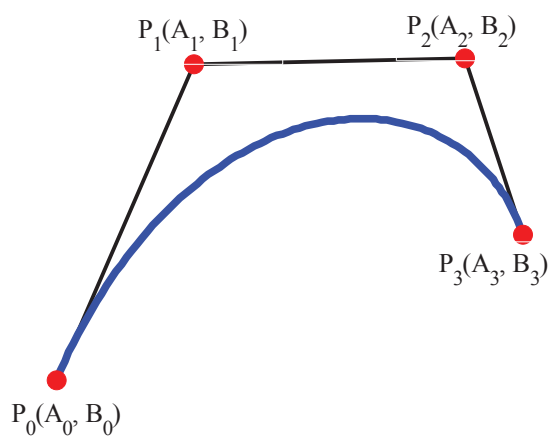

Fig. 2. A cubic Bézier curve defined by four control points

The rest of this paper is organized as follows. Section II gives a brief introduction about the Bézier curve. The door passing strategy is elaborated in Section III, where the problem description, trajectory planning based on Bézier curve as well as the wheelchair control are presented. Section IV presents the simulation results which verify the feasibility of the proposed door passing strategy based on Bézier curves. Finally, a brief conclusion and future work are presented in Section V.

\section{BÉZIER CURVE}

Nowadays, Bézier curve as a powerful and efficient tool has been widely used in computer graphics, animation and fonts because of its capacity of smoothing [12]. The Bézier curves can be formulated as

$$
B(t)=\sum_{i=0}^{n} b_{i, n}(t) P_{i}, \quad t \in[0,1]
$$

where the polynomials

$$
b_{i, n}(t)=\left(\begin{array}{c}
n \\
i
\end{array}\right) t^{i}(1-t)^{n-i}, \quad i=0, \ldots n
$$

are known as Bernstein basis polynomials of degree $n$, and the binomial coefficient, $\left(\begin{array}{c}n \\ i\end{array}\right)$ is expressed as $C_{i}^{n}$. The points $P_{i}$ are called control points for the Bézier curve. The polygon formed by connecting the Bézier points with lines, starting with $P_{0}$ and finishing with $P_{n}$, is called the Bézier polygon (or control polygon). The convex hull of the Bézier polygon contains the Bézier curve. The Bézier curves of degree 1, 2 and 3 are called linear, quadratic and cubic Bézier curves respectively, and quadratic and cubic Bézier curves are most common.

The reasons for why Bézier curves can be ideally used for trajectory planning in robotics rest with the following properties of Bézier curve:

- The curve begins at $P_{0}$ and ends at $P_{n}$. This is the so called endpoint interpolation property.

- The start (end) of the curve is tangent to the first (last) section of the Bézier polygon.

These two properties are exactly the requirements of trajectory planning given two points(start and goal points). The quadratic curve and other curves of higher degree can satisfy with the requirements. However, the quadratic curve is less flexible than cubic curves, and higher degree (more than 3 ) curves are more computationally expensive to evaluate. Therefore, in this paper, to make a balance between flexibility and computational expense of the proposed curve, cubic curves are chosen as the Bézier curves for trajectory planning for the wheelchair to pass the doorway.

According to (1) and (2), the cubic Bézier curve defined by four control points $P_{0}\left(A_{0}, B_{0}\right), P_{1}\left(A_{1}, B_{1}\right), P_{2}\left(A_{2}, B_{2}\right)$ and $P_{3}\left(A_{3}, B_{3}\right)$ in Fig. 2 can be expressed as

$$
\begin{aligned}
B(t) & =\sum_{i=0}^{3} b_{i, n}(t) P_{i}, t \in[0,1] \\
& =(1-t)^{3} P_{0}+3(1-t)^{2} t P_{1}+3(1-t) t^{2} P_{2}+t^{3} P_{3}
\end{aligned}
$$

As we only consider 2-D environment, the cubic Bézier curve can be specified in the form of $B(t)=(x(t), y(t))$ where

$$
\begin{aligned}
& x(t)=A_{0}(1-t)^{3}+3 A_{1}(1-t)^{2} t+3 A_{2}(1-t) t^{2}+A_{3} t^{3} \\
& y(t)=B_{0}(1-t)^{3}+3 B_{1}(1-t)^{2} t+3 B_{2}(1-t) t^{2}+B_{3} t^{3}
\end{aligned}
$$

\section{Door Passing Strategy}

\section{A. Problem Description}

We consider the door passing problem of a wheelchair as a trajectory planning problem. As long as the wheelchair is able to follow the designed path which passes through the doorway without colliding with the door wall as well as other obstacles, then the door passing problem will be well solved. As can be seen in Fig. 3, the wheelchair in the corridor intends to traverse the door on its left side and reach the target in the room. To successfully achieve this goal, the door passing strategy should comply with the following criteria:

- The actual trajectory of the wheelchair has to be graceful and smooth without oscillating or ambiguity, as shown in Fig.3, where the red line represents the desired trajectory in which no obstacles are in the way, and the blue dash line represents the desired trajectory avoiding obstacles.

- Due to the mechanical constraints of the wheelchair, the curvature of the trajectory needs to be limited to certain ranges so that the turning rate will not be too high.

- It is easy for the wheelchair to bump into the door frame if the heading of the wheelchair is not perpendicular to the door plane because of the large volume of the wheelchair. Therefore, it is demanded that the heading of the wheelchair is perpendicular to the door plane when the wheelchair arrives at the $P_{d}$ point.

We propose the general methods for the wheelchair to pass the doorway as: first, dividing the whole trajectory $P_{s} \overparen{P_{d}} P_{t}$ into two parts $-\widetilde{P_{s} P_{d}}$ and $\widetilde{P_{d} P_{t}}$; second, designing the desired trajectory $\widehat{P_{s} P_{d}}$ and $\widehat{P_{d} P_{t}}$ independently based on Bézier curve, to make sure each trajectory meet with the criteria stated above; finally developing an algorithm to control the wheelchair to follow the desired trajectory. 


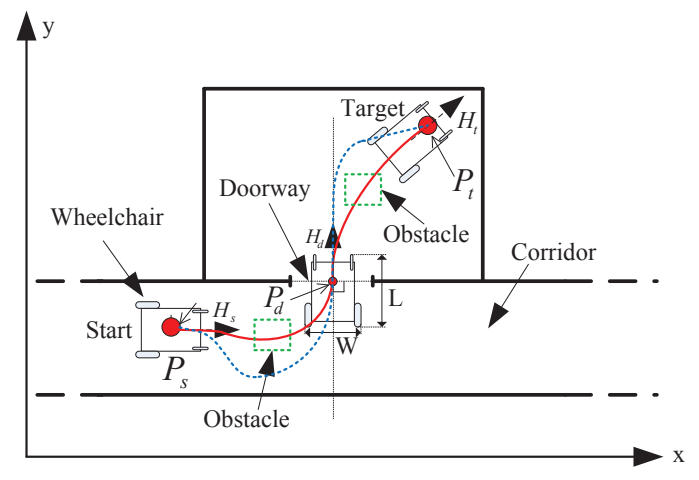

Fig. 3. The schematic description of door passing process of a wheelchair. The red line represents the desired trajectory where no obstacles are in the way, and the blue dash line represents the desired trajectory avoiding obstacles $P_{s}$ represents the current position of the wheelchair, $P_{d}$ is the middle position of the door, and $P_{t}$ is the target position that the wheelchair is supposed to reach.

\section{B. Trajectory Planning based on Bézier Curve}

In this subsection, the trajectory planning for door passing is presented. Its key idea is that the control points which totally define the shape of the Bézier curve are determined using optimization method. The merit of this method lies in the minimum curvature changes of the curve, satisfying the various constraints of the wheelchair and environment and providing the smoothest Bézier curve.

The trajectory planning strategies are divided into two categories depending on whether there is an obstacle. In Fig.3, let $H_{s}, H_{d}$ and $H_{t}$ denote the headings at the positions $P_{s}, P_{d}$ and $P_{t}$ respectively, and denote the control points of $P_{s} P_{d}$ and $\widetilde{P_{d} P_{t}}$ to be $P_{c i}$ and $P_{r i}, i \in 0,1,2,3$, respectively. Then, the trajectory planning is required to generate the cubic Bézier curves which connect the points $P_{s}, P_{d}$ and $P_{t}$ with the orientations $H_{s}, H_{d}$ and $H_{t}$. Because the target in the room is obstructed by the wall, the wheelchair cannot decide the exact position of the target until it closes to the door. Therefore, the whole trajectory $P_{s} \overparen{P_{d}} P_{t}$ has to be divided into two segments $\widetilde{P_{s} P_{d}}$ and $\widetilde{P_{d} P_{t}}$, for which the Bézier curve based trajectories are independently designed.

1) Trajectory Planning without Obstacle: The trajectory planning without obstacle only needs to consider the positions and headings of the beginning and end points of each Bézier curve. There is no demand for obstacle avoidance.

a) Segment from Corridor to Door: When the desired door is detected, the wheelchair uses its current position $P_{s}$, the middle of the door $P_{d}$ and their corresponding orientations to perform the optimization based control point estimation. The door detection can be achieved by some established techniques, such as computer vision. Since $P_{s}$ and $P_{d}$ correspond to the control points $P_{c 0}$ and $P_{c 3}$ respectively, which are known, the parameter estimation only needs to calculate $P_{c 1}$ and $P_{c 2}$, which completely determine the shape of the curve once given $P_{c 0}$ and $P_{c 3}$.

In order to meet the aforementioned requirements, several constraints are devised for the optimization according to the environment and the reality. They are summarized into three types.

i) Corridor structure. In reality, the wheelchair should always be within the corridor while it is turning to the doorway. Therefore, the planned trajectory has to be constrained by the physical structure of the corridor, such as corridor width. Owing to the convex property of Bézier curve, the course can completely lie in the corridor once all the control points are in it. Therefore, the boundary constraint is that $P_{c 1}$ and $P_{c 2}$ can only be chosen from the corridor area. This can be formulated as

$$
P_{c 1} \in C \quad P_{c 2} \in C
$$

where $C$ is the region of the corridor. With this requirement, the produced path can be constrained in the corridor.

ii) Orientations. Since the trajectory planning begins with the heading $H_{s}$, the slope of tangent line of the designed Bézier curve at position $P_{c 0}$ should equal $H_{s}$. Similarly, the tangent line at $P_{c 3}$ should be the normal of the door at the middle in order to overcome the small ratio of door width to the wheelchair's. Therefore, the possible ranges of $P_{c 1}$ and $P_{c 2}$ can be further reduced to one dimension by the means of this idea. In other words, the $P_{c 1}$ and $P_{c 2}$ must respectively locate on the tangent lines at positions $P_{c 0}$ and $P_{c 3}$ due to the aforementioned second property of Bézier curve in Section II. Moreover, $P_{c 1}$ has to be searched along the direction of $H_{s}$, while $P_{c 2}$ only can lie on the opposite direction of $H_{d}$. Hence, the constraints are

$$
\begin{aligned}
& \theta_{0}=\arccos \left(\frac{\overrightarrow{P_{c 0} P_{c 1}} \cdot H_{s}}{\left\|\overrightarrow{P_{c 0} P_{c 1}}\right\| \cdot\left\|H_{s}\right\|}\right)=0 \\
& \theta_{3}=\arccos \left(\frac{\overrightarrow{P_{c 2} P_{c 3}} \cdot H_{d}}{\left\|\overrightarrow{P_{c 2} P_{c 3}}\right\| \cdot\left\|H_{d}\right\|}\right)=0
\end{aligned}
$$

These restrictions ensure that the Bézier curve determined by $P_{c 1}$ and $P_{c 2}$ can join the starting and end points $P_{c 0}$ and $P_{c 3}$ with the desired orientations $H_{s}$ and $H_{d}$.

iii) Complete convexity. The curvature limitation described in III-A requires that there is no sharp bend or sudden change of curvature in the curve. Thus, the Bézier curve is better to be completely convex. It has been known that a Bézier curve is completely convex if its control polygon is convex. Therefore, $P_{c 1}$ and $P_{c 2}$ have to be constrained to guarantee the complete convexity of the curve. The cross product method is introduced in this proposed strategy to fulfill this convex requirement. The cross products $\omega_{1}$ and $\omega_{2}$ of vectors $\overrightarrow{P_{c 0} P_{c 1}}, \overrightarrow{P_{c 1} P_{c 2}}$ and $\overrightarrow{P_{c 2} P_{c 3}}$ are

$$
\begin{aligned}
& \omega_{1}=\overrightarrow{P_{c 0} P_{c 1}} \times \overrightarrow{P_{c 1} P_{c 2}} \\
& \omega_{2}=\overrightarrow{P_{c 1} P_{c 2}} \times \overrightarrow{P_{c 2} P_{c 3}}
\end{aligned}
$$

Therefore, the constraint of the complete convexity is

$$
\operatorname{sign}\left(Z_{\omega_{1}}\right) \cdot \operatorname{sign}\left(Z_{\omega_{2}}\right)>0
$$

where $\operatorname{sign}\left(Z_{\omega_{i}}\right), i=1,2$, are the rotation direction of the $\omega_{i}$. 
The constrained optimization problem is to find the control points $P_{c 1}$ and $P_{c 2}$ which make the curve smooth. Then, the curvature and the rate of its change should be as small as possible. According to (4), the curvature of a Bézier curve with respect to $t$ is

$$
\kappa(t)=\frac{1}{\rho(t)}=\frac{\dot{x}(t) \ddot{y}(t)-\dot{y}(t) \ddot{x}(t)}{\left(\dot{x}^{2}(t)+\dot{y}^{2}(t)\right)^{3 / 2}}
$$

where $\rho(t)$ is the radius of curve. Therefore, $P_{c 1}$ and $P_{c 2}$ can be computed by the following constrained optimization problem, which is subjected to (5), (6) and (8):

$$
\begin{array}{cc}
\min _{P_{c 1}, P_{c 2}} & \int_{0}^{1}\left[(\kappa(t))^{2}+(\dot{\kappa}(t))^{2}\right] d t \\
\text { s.t. } & \text { (5) (6) (8) }
\end{array}
$$

where $\dot{\kappa}(t)$ is the first derivative of curvature. The interior point method is introduced in this study to solve all the optimization problems. Thus, the calculated $P_{c 1}$ and $P_{c 2}$ can meet the various requirements and provide an optimal trajectory planning for the first part $\widehat{P_{s} P_{d}}$.

b) Segment from Door to Target: When the wheelchair arrives in the doorway, it is expected to be at the center of the door with heading being perpendicular to the door. However, because of the disturbances and the control errors, the final position and orientation of last step may not coincide with the desired ones even though the errors may be small under an efficient control strategy. In order to satisfy the third requirement in III-A, the true heading $H_{d}^{a}$ needs to be adjusted to the perpendicular direction of the door before addressing the trajectory planning $\widetilde{P_{d} P_{t}}$.

This trajectory planning from door to target should start from the true position $P_{d}^{T}$ with the heading $H_{d}^{a}$ in order to maintain the smoothness of the intersection of the two Bézier curves. Moreover, the $P_{d}^{T}$ and $P_{t}$ correspond to the control points $P_{r 0}$ and $P_{r 3}$ of the Bézier curve respectively. The main idea of this trajectory planning from door to target is similar to the segment from corridor to door except some specified constraints. The constrained optimization problem of this part is

$$
\begin{array}{cc}
\min _{P_{r 1}, P_{r 2}} & \int_{0}^{1}\left[(\kappa(t))^{2}+(\dot{\kappa}(t))^{2}\right] d t \\
\text { s.t. } & P_{r 1} \in R \quad P_{r 2} \in R \\
& \arccos \left(\frac{\overrightarrow{P_{r 0} P_{r 1}} \cdot H_{d}^{a}}{\| \overrightarrow{P_{r 0} P_{r 1}\|\cdot\| H_{d}^{a} \|}}\right)=0 \\
& \arccos \left(\frac{\overrightarrow{P_{r 2} P_{r 3}} \cdot H_{t}}{\| \overrightarrow{P_{r 2} P_{r 3}\|\cdot\| H_{t} \|}}\right)=0 \\
& \operatorname{sign}\left(Z_{\omega_{3}}\right) \cdot \operatorname{sign}\left(Z_{\omega_{4}}\right)>0
\end{array}
$$

where $R$ is room area, and $Z_{\omega_{i}}, i=3,4$, are the cross products of vectors $\overrightarrow{P_{r 0} P_{r 1}}, \overrightarrow{P_{r 1} P_{r 2}}$ and $\overrightarrow{P_{r 2} P_{r 3}} .(11 \mathrm{~b})$ is the room boundary constraint, which bounds the planned trajectory by setting the the control points $P_{r 1}$ and $P_{r 2}$ in the room area. As the orientation and complete convexity constraints, (11c), (11d) and (11e) are respectively very similar to $(6 a),(6 b)$ and (8) in terms of format and functions. Since the initial value of the optimization problem highly decides if

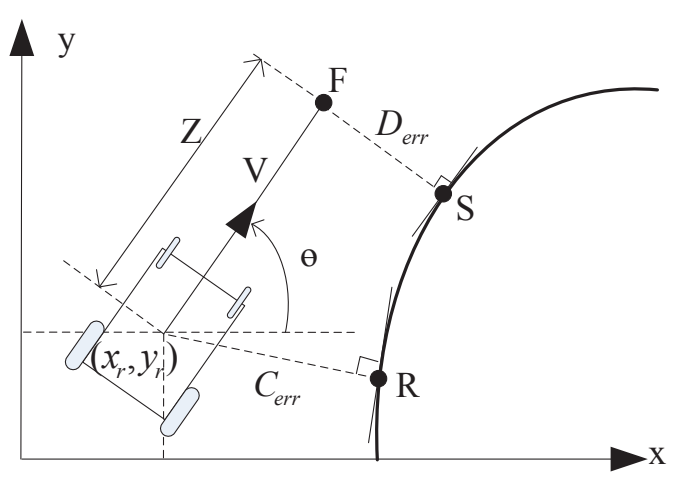

Fig. 4. The position error of the wheelchair is calculated from a point $F$, which is projected in front of the vehicle with a distance of $t$, and unto the desired trajectory to point $S$ [11].

the global minimum can be reached, the beginning point of the period $P_{d}^{T}$ is used as the initialization for $P_{r 1}$ and $P_{r 2}$.

2) Trajectory Planning with Obstacle: It is well-known that the door crossing is more difficult for the wheelchair with an obstacle around and this scene is common in practice. In general, the typical techniques divide the door crossing problem into two phases: first, the wheelchair is driven to the area near the door, avoiding the obstacle; then, the door crossing strategy is designed to pass the doorway successfully. However, the special obstacle avoidance step is redundant, especially for the static obstacle, when a one step method is adopted to efficiently address the door passing with obstacle.

This approach also transforms the door crossing with obstacle into the optimization problem, and it is mainly based on the aforementioned trajectory planning. However, the planning of the Bézier curve has to be adjusted according to the obstacle. Assume the static obstacles are distributed as Fig.3. Then, the problem can be addressed by adding an additional constraint to the optimization problem (10) and (11):

$$
\begin{array}{r}
\left(y\left(t_{k}\right)-y_{o}\right)^{2}+\left(x\left(t_{k}\right)-x_{o}\right)^{2}>d_{r}^{2} \\
t_{k} \in[0,1], k \in[1,1000]
\end{array}
$$

where $x\left(t_{k}\right)$ and $y\left(t_{k}\right)$ are the discrete points sampled from (4), and $d_{r}$, which depends on the dimensions of the wheelchair and the obstacle, is the allowable minimum distance between the wheelchair and the obstacle. The computed control points $P_{c 1}, P_{c 2}, P_{r 1}$ and $P_{r 2}$ under the above constraint can provide the desired pathes for the wheelchair to pass the door with obstacle avoidance.

\section{Wheelchair Control}

In order to drive the wheelchair to track the desired trajectory, a feedback control principle using PID controller is adopted. The position error between the actual position of the wheelchair and the reference trajectory is used as the input of the PID controller. As can be seen in Fig.4, and a point $F$ ahead along the heading of the wheelchair with a distance of $Z$ is used to define the position error, $F$ is projected onto the 
TABLE I

THE INPUT PARAMETERS FOR EACH TRAJECTORY

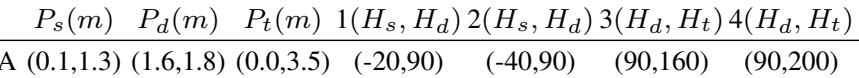

B $(0.1,1.3)(1.6,1.8)(3.4,3.5) \quad(-10,90) \quad(10,90) \quad(90,20) \quad(90,-20)$

reference trajectory at point $S$ such that $\overline{F S}$ is perpendicular to the tangent at $S$. The position error is then represented by the distance $D_{\text {err }}$ between point $F$ and $S$. The cross track error $C_{e r r}$ is defined by the shortest distance between the desired trajectory and the position of the center of the gravity of the wheelchair $\left(x_{r}, y_{r}\right)$, and $C_{e r r}$ will be adopted as the indication of the control performance in the simulation analysis in Section IV.The position of the wheelchair $\left(x_{r}, y_{r}\right)$ is assumed to be estimated by other techniques such as SLAM algorithms.

In this paper, the longitudinal velocity $V$ is considered as constant, and the angular rate $\omega$ as the control output of the PID controller. The discretized PID controller can be expressed as

$$
\omega=k_{p} D_{e r r}^{k}+k_{i} T_{s} \sum_{i=1}^{k} D_{e r r}^{i}+\frac{k_{d}}{T_{s}}\left(D_{e r r}^{k}-D_{e r r}^{k-1}\right)
$$

where $k_{p}, k_{i}$ and $k_{d}$ are proportional, integral and derivative gains respectively, $D_{e r r}^{k}$ is the current position error, and $D_{e r r}^{k-1}$ is the position error of last time instance. The reason for why $D_{\text {err }}$ rather than $C_{\text {err }}$ is used as the input of the PID controller lies in the fact that the required $k_{p}$ for compensating the same quantity of $C_{e r r}$ is much larger than that of $D_{e r r}$, and the larger $k_{p}$ is more likely to cause system oscillation.

\section{Simulation Results}

In order to verify the proposed Bézier curve door passing strategy, simulation work has been conducted. First we carried out two simulation scenarios, namely Scenario A and B, where no obstacles are considered. For each scenario, four different reference trajectories are determined by our Bézier curve based trajectory planning algorithms using optimization technique. The parameters such as the starting position of the wheelchair and its heading, the target position and the heading should be given for determining each reference trajectory.

The required parameters for each reference trajectory are listed in Table I, where $P_{s}, P_{d}$ and $P_{t}$ represent the coordinate of starting point in corridor, the coordinate of the middle of the door and the coordinate of the target point in the room respectively. $1\left(H_{s}, H_{d}\right)$ represents the heading of the starting position and the end position for trajectory 1 , and the same meaning goes with other $i\left(H_{s}, H_{d}\right)$ or $i\left(H_{d}, H_{t}\right)$ with $i$ being the $i$ th trajectory. Using these parameters as the inputs, the Bézier curve based trajectory planning strategy is able to calculate the optimized smooth reference trajectory for the wheelchair to follow gracefully. The PID controller, whose gains are selected to be $k_{p}=0.01, k_{i}=0.001$ and $k_{d}=0.001$, is then adopted to drive the wheelchair to track the reference trajectory.

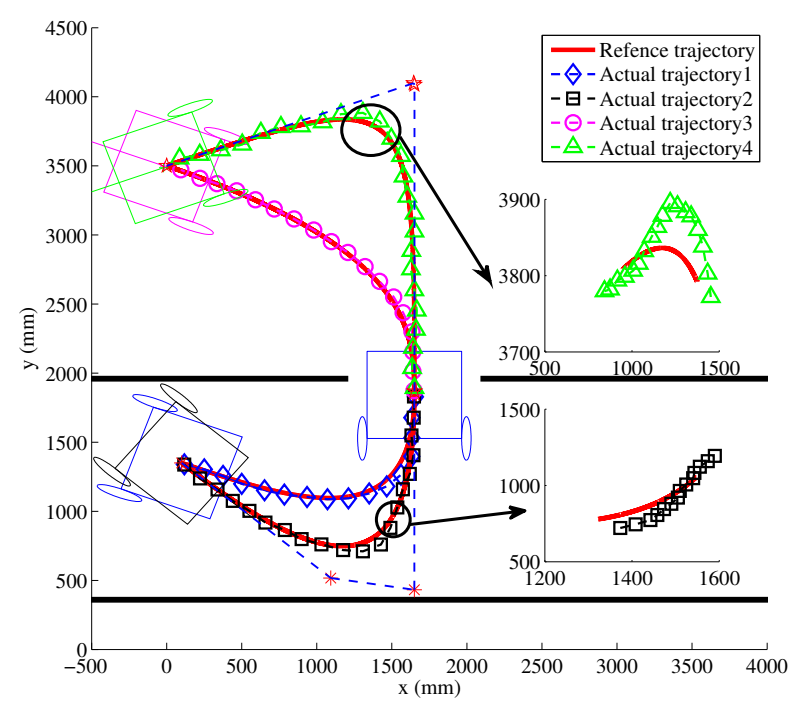

(a) Scenario A

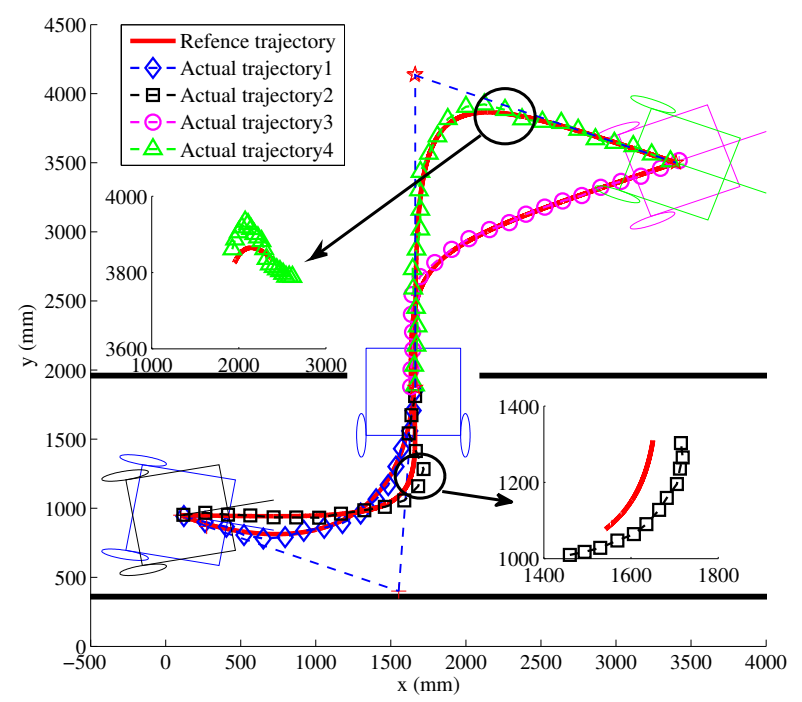

(b) Scenario B

Fig. 5. Reference trajectory and actual trajectory of wheelchair.

Fig. 5 shows the calculated reference trajectories and their corresponding actual trajectories of the wheelchair given the parameters in Table I. As can be seen, several conclusions can be drawn: 1) All the optimized reference trajectories satisfy with the constraints mentioned in Section III-B. That is: first, the wheelchair always is within the corridor; second, the tangent of the Bézier curve at the starting position is equal to the heading of the wheelchair, and the same situation goes with the target position; the control polygon of the designed Bézier curve is convex. 2) The optimized reference trajectories are smooth. 3) The actual trajectories precisely track the reference trajectory, although there are small tracking errors (can be seen from the amplified part of trajectories). With this fact, the basic 


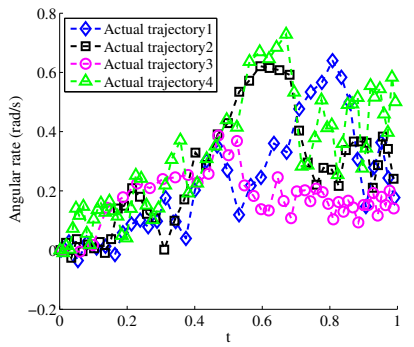

(a) Angular rate

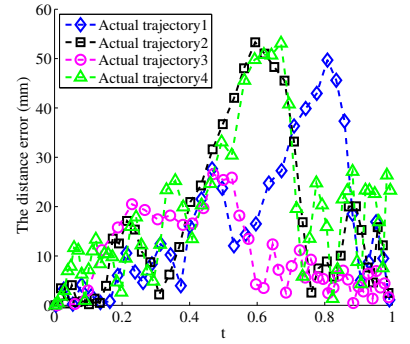

(b) Tracking error
Fig. 6. The angular rate and the tracking errors for each trajectory in Scenario A

goal of door passing is successfully achieved.

To analyze the smoothness and the accuracy of the actual trajectories that the wheelchair follows, the angular rate $\omega$ and the distance error $D_{e r r}$ with respect to the Bézier curve parameter $t$ for each trajectory in Scenario A are shown in Fig. 6a and Fig. 6b respectively. It can be seen that there is no sharp change of the angular rate, which demonstrates the wheelchair is able to move smoothly. The maximum tracking error is about $55 \mathrm{~mm}$, which is both reasonable and acceptable, indicating the relatively high accuracy of the tracking. In order to validate the scheme with obstacle, a scenario where two obstacles are separately distributed in the corridor and room is simulated, see Fig. 7. The blue circles are obstacles, and the widths of wheelchair, corridor and door are assumed to be 75,160 and 90 centimeters respectively. Compared the trajectory planning without obstacle, which is denoted by the red dash line, with the one affected by the obstacle, which is represented by the diamond and square dash lines, it can be seen that the wheelchair can dynamically adjust its planned trajectories according to the position of the obstacle, avoiding the obstacles and still reaching the destination smoothly.

\section{Conclusions}

In this paper, a novel method is proposed to address the tough door crossing problem of the wheelchair. It introduces the Bézier curve based trajectory planning and optimization to produce a smooth and reasonable reference trajectory for the wheelchair to follow. Its merit is that it sufficiently considers the various constraints of the wheelchair and the environment, and can perform the door crossing with graceful and smooth trajectories even hindered by the obstacles. The good performance of the proposed approach is also verified by the means of simulation. It is not limited to the wheelchair, but also can be used for the various mobile robots which intend to pass the narrow door. Our future work will focus on implementing the proposed approach on a real wheelchair.

\section{ACKNOWLEDGMENTS}

The authors gratefully acknowledge the support of the UK EPSRC Global Engagements grant EP/K004638/1 and the EU Interreg IV A 2 Mers Seas Zeen Cross-border Cooperation Programme SYSIASS project: Autonomous and Intelligent

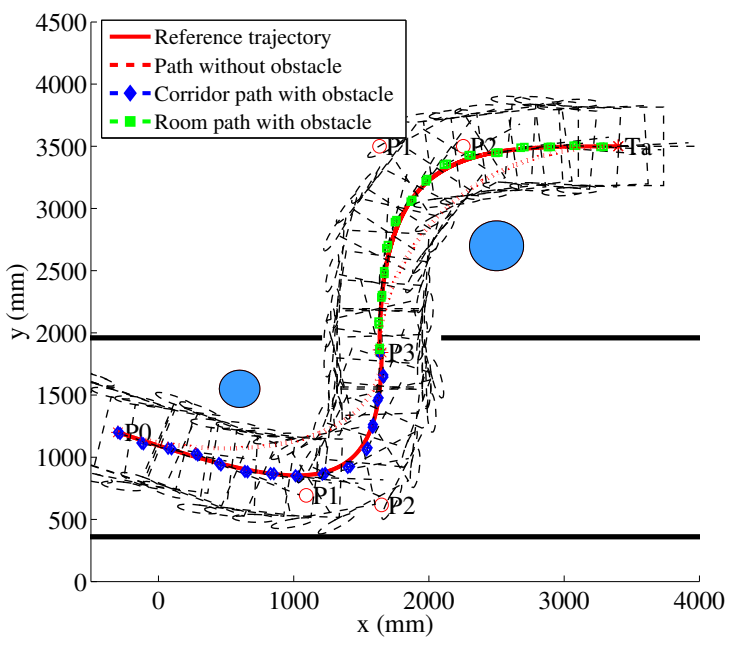

Fig. 7. Trajectories of the with and without obstacles.

Healthcare System (http://www.sysiass.eu/). Ling Chen and Sen Wang have been financially supported by scholarships from China Scholarship Council and Essex University. Our thanks also go to Robin Dowling for his technical support during the research.

\section{REFERENCES}

[1] O. Khatib, "Real-time obstacle avoidance for manipulators and mobile robots," The international journal of robotics research, vol. 5, no. 1, pp. 90-98, 1986.

[2] J. Barraquand, B. Langlois, and J. Latombe, "Numerical potential field techniques for robot path planning," IEEE Transactions on Systems, Man and Cybernetics, vol. 22, no. 2, pp. 224-241, 1992.

[3] F. Arambula Cosío and P. Castañeda, "Autonomous robot navigation using adaptive potential fields," Mathematical and computer modelling, vol. 40, no. 9-10, pp. 1141-1156, 2004.

[4] R. Simpson, "Smart wheelchairs: A literature review." Journal of rehabilitation research and development, vol. 42, no. 4, p. 423, 2005.

[5] BSI, Design of buildings and their approaches to meet the needs of disabled people-Code of practice, British Standards Institution. Std. BS 3800, 2009.

[6] F. Cheein, C. De La Cruz, T. Bastos, and R. Carelli, "Slam-based crossa-door solution approach for a robotic wheelchair," International Journal of Advanced Robotic Systems, vol. 7, no. 2, pp. 155-164, 2010.

[7] A. Poncela, C. Urdiales, and F. Sandoval, "A cbr approach to behaviourbased navigation for an autonomous mobile robot," in 2007 IEEE International Conference on Robotics and Automation. IEEE, 2007, pp. 3681-3686.

[8] A. Scheuer and T. Fraichard, "Continuous-curvature path planning for car-like vehicles," in Proceedings of the 1997 IEEE/RSJ International Conference on Intelligent Robots and Systems, 1997., vol. 2. IEEE, 1997, pp. 997-1003.

[9] J. Connors and G. Elkaim, "Analysis of a spline based, obstacle avoiding path planning algorithm," in IEEE 65th Vehicular Technology Conference. IEEE, 2007, pp. 2565-2569.

[10] K. Jolly, R. Sreerama Kumar, and R. Vijayakumar, "A bezier curve based path planning in a multi-agent robot soccer system without violating the acceleration limits," Robotics and Autonomous Systems, vol. 57, no. 1, pp. 23-33, 2009.

[11] J. Choi, R. Curry, and G. Elkaim, "Path planning based on bézier curve for autonomous ground vehicles," in Advances in Electrical and Electronics Engineering-Special Edition of the World Congress on Engineering and Computer Science. IEEE, 2008, pp. 158-166.

[12] J. Foley, A. Van Dam, S. Feiner, J. Hughes, and R. Phillips, Introduction to computer graphics. Addison-Wesley, 1994, vol. 55. 